\title{
THE FIRST SUMMER MATHEMATICAL INSTITUTE
}

The First Summer Mathematical Institute, devoted to Lie algebras and Lie groups, was held from June 20 to July 31, 1953 at Colby College, Waterville, Maine. It was supported by a grant from the National Science Foundation to the American Mathematical Society. The Organization Committee consisted of C. C. Chevalley, A. M. Gleason, and Nathan Jacobson (chairman).

The following twenty mathematicians attended the Institute by invitation of the Organization Committee: Armand Borel, C. C. Chevalley, W. L. Chow, A. M. Gleason, Morikumi Goto, G. P. Hochschild, Kenkichi Iwasawa, Nathan Jacobson, Irving Kaplansky, ${ }^{1}$ E. R. Kolchin, W. G. Lister, Deane Montgomery, ${ }^{1}$ G. D. Mostow, Hans Samelson, R. D. Schafer, E. V. Schenkman, H. C. Wang, Hidehiko Yamabe, Hans Zassenhaus, and Leo Zippin. ${ }^{1}$

The Institute was open to all interested mathematicians and the following nine attended: S. A. Amitsur, S. G. Bourne, J. L. Brenner, H. E. Campbell, C. W. Curtis, E. C. Paige, Jr., A. J. Penico, G. B. Seligman, and M. L. Tomber.

The formal program of the Institute consisted of a seminar on simple Lie algebras and the following four series of lectures:

Armand Borel, The cohomology of compact connected Lie groups and their coset spaces;

C. C. Chevalley, Cartan subalgebras and Cartan subgroups;

Hidehiko Yamabe, Structure of locally compact groups;

Hans Zassenhaus, Representation theory of Lie algebras of characteristic $p$.

These are summarized below.

In addition there was a series of single talks on various mathematical topics as follows:

Kenkichi Iwasawa, On some totally disconnected compact groups.

H. C. Wang, Closed manifolds with homogeneous complex analytic structure.

Morikumi Goto, Dense imbeddings of locally compact connected groups.

E. R. Kolchin, Galois theory of differential fields.

W. L. Chow, The Albanese variety of an algebraic variety.

A. M. Gleason, Lattices of topologies.

Hans Zassenhaus, Trace functions of characteristic $p$.

G. D. Mostow, Uniform subgroups of solvable groups.

Received by the editors June 7, 1954.

1 Attended for the first three weeks only. 
C. C. Chevalley, Galois theory of semi-simple Lie algebras.

Colby College provided office space and dormitory accommodations for the members of the Institute and their families and made available their recreation area on Great Pond where a picnic was held on the fourth of July.

Beyond the lectures themselves, the informal contacts that the Institute afforded were tremendously valuable. Several papers, many of them collaborations, will soon appear in a Memoirs volume enentitled Proceedings of the First Summer Mathematical Institute, and it is expected that many other results will evolve from the stirring of ideas effected by the six weeks of study and conversation.

\section{A. M. Gleason}

\section{THE COHOMOLOGY OF COMPACT CONNECTED LIE GROUPS AND THEIR COSET SPACES}

\section{Lectures by Armand Borel}

This seminar was devoted to a survey of the main results obtained so far in the study of the cohomology ring of compact connected Lie groups and their homogeneous spaces. One may roughly divide these twelve talks into four groups.

In the first part were discussed general properties of groups, i.e., properties which derive solely from the existence of a product (say associative with unit); that such an approach is fruitful was shown by $\mathrm{H}$. Hopf, who proved that the cohomology ring $H^{*}\left(X, K_{0}\right)$ of such a compact manifold, with respect to a field of coefficients $K_{0}$ of characteristic zero, is a Grassmann algebra generated by elements of odd degrees, or in other words that it is isomorphic to the cohomology ring of a product of odd-dimensional spheres. A generalization of this theorem, valid for coefficient fields of any characteristic, was established. The product in $X$ also allows one to define a product (introduced by Pontrjagin) in the homology group of $X$, which thus becomes a ring; over a field of characteristic zero, it is again an exterior algebra (Samelson's theorem), but this result has been generalized only in some special cases; the chief new difficulty arising here is the fact that the Pontrjagin product does not have the familiar anticommutativity property of the cup-product in general.

The second part was devoted to relations between the cohomology ring $H^{*}\left(G, K_{p}\right)\left(K_{p}\right.$ field of characteristic $\left.p\right)$ of a compact connected Lie group $G$ and the cohomology ring $H^{*}\left(B_{G}, K_{p}\right)$ of a classifying space $B_{G}$ for $G$, a point which is at the source of most of the progress made recently in these questions. We recall that a fiber bundle $E$, 
with base $B$ and typical fiber $F$, is said to be a principal bundle with structural group $G$ if $G$ operates on $E$, leaving each fiber invariant, and being simply transitive on them (thus $F$ is homeomorphic to $G$ ); it is a universal bundle for $G$ and for $n$ if moreover its $n$ first homotopy groups vanish. The existence of universal bundles for any compact Lie group and any $n$ is well known and important. The base space of a universal bundle is called a classifying space for $G$ (and for $n$ ), and denoted $B_{G}$; it owes its name to the fact that the different structures of principal bundles with structural group $G$ over a given locally finite polyhedron $B$ (of dimension $\leqq n$ ) are in 1-1 correspondence with the homotopy classes of continuous maps of $B$ into $B_{G}$. By use of Leray's spectral sequence of fiber bundles one can establish relations between $H^{*}\left(G, K_{p}\right)$ and $H^{*}\left(B_{G}, K_{p}\right)$, or more precisely one can see how certain assumptions on the structure of one of them affect the other; that study has not yet been performed in the most general case; however, partial results (with a rather wide range of applications) have been obtained and were discussed. The main one says that if $H^{*}\left(G, K_{p}\right)$ is an exterior algebra generated by $r$ elements of odd degrees $r_{i}(1 \leqq i \leqq m)$, then $H^{*}\left(B_{G}, K_{p}\right)$ is a ring of polynomials over $r$ variables of degrees $r_{i}+1$, and conversely.

When $U$ is a closed subgroup of $G$, there exists a natural map of $B_{U}$ into $B_{G}$, denoted $\rho(U, G)$, which is particularly interesting when $U=T^{r}$ is a maximal torus of $G$; in that case $H^{*}\left(B_{U}, K_{p}\right)$ is a ring of polynomials in $r$ variables of degree two, on which the Weyl group of $G$ acts in a natural fashion (e.g., over the real field, $H^{*}\left(B_{T^{r}}, R\right)$ may be identified with the ring of polynomials with real coefficients on the Lie algebra of $T^{r}$ ). It was shown that under suitable assumptions, always fulfilled in characteristic zero (mainly $H^{*}(G, Z)$ has no $p$-torsion, i.e., has no torsion coefficient divisible by $p$ ), the homomorphism $\rho^{*}\left(T^{r}, G\right)$ induced by $\rho\left(T^{r}, G\right)$ maps $H^{*}\left(B_{G}, K_{p}\right)$ isomorphically into $H^{*}\left(B_{T^{r}}, K_{p}\right)$, essentially on the ring of invariants of the Weyl group. Thus one gets a group-theoretical interpretation of $H^{*}\left(B_{G}, K_{p}\right)$, which, via the theorem stated at the end of the preceding paragraph, also gives relations between $H^{*}\left(G, K_{p}\right)$ and the Weyl group (obtained first in characteristic zero by $C$. Chevalley). Whether one can tie up so closely $H^{*}\left(B_{G}, K_{p}\right)$ with group theoretical properties of $G$ in the general case where $G$ has $p$-torsion is unknown; modulo 2, however, a special case has been worked out, that of the orthogonal group, where replacing the maximal torus by the subgroup of diagonal matrices leads to results bearing a close and suggestive analogy to the preceding ones.

The methods and results expounded in the first two parts do not 
allow one in general to determine $H^{*}\left(G, K_{p}\right)$ for a given $G$ having $p$ torsion, and for that purpose one has to use special devices, taking advantage of some particular properties of the group in consideration. The third group of talks described how the above general theorems combined with the study of spectral sequences of special fiberings give the cohomology mod $p$ of the classical groups, the spinor groups, and the first two exceptional groups $G_{2}, F_{4}$.

The last part of the seminar was devoted to homogeneous spaces. Here the problem is to find relations between the cohomology rings of a group $G$, a subgroup $U$, and the coset space $G / U$; to be more specific, one wants mainly to determine the last one by means of the two others and of some information describing the position of $U$ in $G$. Rather complete results in that direction have been obtained for the cohomology over the real numbers, by use of differential-geometric and algebraic methods, the most comprehensive one being a theorem of H. Cartan; it shows how to calculate (at least theoretically) $H^{*}(G / U, R)$, knowing $H^{*}(G, R)$, and certain data which turn out to be essentially $H^{*}\left(B_{U}, R\right)$ and $\rho^{*}(U, G)$, and there was given here a topological proof, in the framework of fiber bundle theory.

Mod $p$, the situation is less satisfactory; though the methods and results known so far are sufficient to deal with many classical homogeneous spaces, they nevertheless pertain only to special cases. The discussion was restricted mainly to one of these, that of equality of ranks (i.e., $G$ and $U$ have a maximal torus in common); also, the analogy mentioned previously between the role of maximal tori in real cohomology and of the diagonal matrices for the cohomology mod 2 of the orthogonal group $O(n)$ was pursued further and allowed us to determine $H^{*}\left(O(n) / U, K_{2}\right)$ when $U$ has the same "rank mod 2" as $O(n)$, i.e., when it contains an abelian subgroup of type $(2, \cdots, 2)$ maximal in $O(n)$.

ARMAND Borel

\section{Cartan subalgebras and Cartan subgroups}

\section{Lectures by C. C. Chevalley}

We consider a field $K$ of characteristic zero and a vector space $V$ of finite dimension $n$ over $K$. The set $L(V)$ of all $K$-endomorphisms of $V$ is a vector space of dimension $n^{2}$ over $K . L(V)$ becomes a Lie algebra if it is endowed with the bracket operation $[X, Y]=X Y$ $-Y X$; we denote this Lie algebra by $\mathfrak{g l}(V)$. If the intersection of of some algebraic variety in $L(V)$ with the group of nonsingular endomorphisms of $V$ is a group, then $\$$ is is called an algebraic group of 
automorphisms of $V$. Forming the differentials (formal) at the unit element of the polynomials defining (\$), we get a family of linear functions on $\mathfrak{g l}(V)$ which annihilate a subalgebra of $\mathfrak{g l}(V)$ called the Lie algebra of 5 . Not every subalgebra of $\mathfrak{g l}(V)$ arises in this way; those that do are called algebraic Lie algebras. Algebraic groups and their Lie algebras can now be studied by the methods of algebraic geometry.

A subalgebra $\mathfrak{h}$ of a Lie algebra $\mathfrak{g}$ is said to be nilpotent if, for each $X \in \mathfrak{h}$, the endomorphism $Y \rightarrow[X, Y]$ of $\mathfrak{g}$ is nilpotent. The normalizer of a subalgebra $\mathfrak{h}$ is the largest subalgebra of $\mathfrak{g}$ in which $\mathfrak{h}$ is an ideal. We define a Cartan subalgebra $\mathfrak{h}$ of $\mathfrak{g}$ as a nilpotent subalgebra of $\mathfrak{g}$ which is its own normalizer.

A group is nilpotent if the ascending central series terminates with the whole group. The subgroup $\mathfrak{S}$ of $\mathbb{S}$ is called a Cartan subgroup if $\mathfrak{S}$ is a maximal nilpotent subgroup of $\mathbb{S}$ and if every normal subgroup $\mathfrak{R}$ of finite index in $\mathfrak{S}$ is of finite index in the normalizer of $\mathfrak{R}$.

The main objective of the lectures was the proof of the following theorem.

Let $\$$ be an irreducible algebraic group and let $g$ be the Lie algebra of $\mathbb{B}$. If $\mathfrak{S}$ is a Cartan subgroup of $\mathbb{G}$, then $\mathfrak{S}$ is algebraic and its Lie algebra is a Cartan subalgebra of $\mathfrak{g}$. If $\mathfrak{h}$ is a Cartan subalgebra of $\mathfrak{g}$, then it is the Lie algebra of some Cartan subgroup of $\$$ ).

\section{A. M. GLeason}

\section{THE STRUCTURE OF LOCALLY COMPACT GROUPS}

\section{Lectures by Hidehiko Yamabe}

The main objective in the theory of locally compact groups is to relate them to Lie groups. The structure theorem can be stated as follows:

THEOREM. Let $G$ be a connected locally compact group. In every neighborhood of the identity there is a compact normal subgroup $N$ such that $G / N$ is a Lie group.

While this result is not valid for disconnected locally compact groups, every locally compact group contains an open subgroup for which the above conclusion holds. This fact is easily deduced from the theorem as stated.

The theorem was first proved for groups of finite topological dimension by Montgomery and Zippin [3]. Yamabe [5; 6] has proved the general case using a refinement of a technique introduced by Gleason 
[1] in proving that finite-dimensional locally compact groups without small subgroups are Lie groups.

The reasoning given by von Neumann [4] in his solution of Hilbert's fifth problem for compact groups in conjunction with this theorem readily proves the affirmative answer to the customary form of Hilbert's fifth problem: Every locally Euclidean group is a Lie group.

Yamabe begins with a connected locally compact group $G$ and proves that in any neighborhood of the identity there is a compact normal subgroup $N$ for which $G / N$ has no small subgroups; i.e., there is a neighborhood $V$ of the identity in $G / N$ which contains no entire nontrivial subgroup. The remainder of the proof consists in showing that $G / N$ is a Lie group.

Starting once again with a connected locally compact group $H$ having no small subgroups, he constructs a linear space $T$ of "tangent vectors to $H^{\prime \prime}$ in $L^{2}(H)$, the Hilbert space of square integrable functions with respect to the Haar measure of $H$, and a homeomorphism of a neighborhood of the origin in $T$ onto a compact subset of $G$. (This turns out to be the exponential mapping when $T$ is identified with the Lie algebra of $G$.) The existence of such a homeomorphism proves that $T$ has finite dimension. The inner automorphisms of $H$ induce linear transformations on $T$; this gives a finite-dimensional representation of $H$ whose kernel is just the center $C$. (This is the adjoint representation in the classical theory of Lie groups.) By von Neumann's theorem $H / C$ is a Lie group. Since $C$ is a locally compact abelian group without small subgroups, it is already known to be a Lie group from the work of Pontrjagin. At this point one can apply the theorem of Kuranishi [2] and deduce that $H$ is a Lie group, but, since Kuranishi's proof depends on long arguments with differential equations, it is more satisfying to prove that $H$ is a Lie group directly. This Yamabe does by explicitly calculating the group multiplication in terms of the canonical coordinates induced in $H$ by the exponential mapping of the tangent vectors.

\section{REFERENCES}

1. A. M. Gleason, Groups without small subgroups, Ann. of Math. vol. 56 (1952) pp. 193-212.

2. Masatake Kuranishi, On Euclidean local groups satisfying certain conditions, Proc. Amer. Math. Soc. vol. 1 (1950) pp. 372-380.

3. Deane Montgomery and Leo Zippin, Small subgroups of finite-dimensional groups, Ann. of Math. vol. 56 (1952) pp. 213-241.

4. J. von Neumann, Die Einführung analytischer Parameter in topologischen Gruppen, Math. Ann. vol. 34 (1933) pp. 170-190. 
5. Hidehiko Yamabe, On the conjecture of Irwasawa and Gleason, Ann. of Math. vol. 58 (1953) pp. 48-54.

6. - A generalization of a theorem of Gleason, Ibid. pp. 351-365.

\section{A. M. Gleason}

\section{REPRESENTATION THEORY OF LIE ALGEBRAS OF CHARACTERISTIC $p$}

\section{Lectures by Hans Zassenhaus}

1. Introduction. The first serious investigations of the linear representations of Lie algebras of prime characteristic were carried out by Zassenhaus [10], who made an exhaustive study of the irreducible representations of nilpotent Lie algebras, and by Ho-Jui Chang [3], who determined the irreducible representations of the Witt algebras. At about the same time, Jacobson began his work on restricted Lie algebras of characteristic $p>0$, and proved a number of important theorems concerning their representations. These results give many indications of the shape a general theory must assume, but such a theory has been slow to materialize. Nevertheless, important results of a general nature have been obtained recently by Jacobson and Zassenhaus.

Jacobson's contribution, to be found in [7], included the observation that the universal associative algebra $\mathfrak{A}$ of a Lie algebra $\mathfrak{R}$ over a field of characteristic $p>0$ is a finitely generated module over its center. This result led to a new and simple proof of Iwasawa's theorem that $\mathbb{R}$ has a faithful finite-dimensional representation, a proof of a conjecture of Chevalley which asserts that every Lie algebra of prime characteristic has a representation which is not completely reducible, and a proof of the fact that $\mathfrak{A}$ can be imbedded in a division algebra, finite-dimensional over its center.

By exploiting the fact that $\mathfrak{A}$ can be imbedded in a division ring, Zassenhaus exhibited a bound on the degree of the absolutely irreducible representation (a.i.r.) of $\mathfrak{R}$, and proved that the maximum degree was assumed except for those a.i.r. which annihilate a certain discriminant ideal associated with $\mathfrak{O}$. His methods also lead to the theorem that $\mathfrak{R}$ has indecomposable representations of arbitrarily high degree.

This report contains a more detailed description of these results, together with some comments on the proofs. A few unsolved problems have also been listed.

2. The universal associative algebra. We begin with the result of Birkhoff [1] and Witt [9], which asserts that a Lie algebra $\mathbb{R}$ over an arbitrary field $K$ has a faithful imbedding $\eta$ in an associative algebra 
$\mathfrak{I}^{*}$, which is universal in the sense that if $\sigma$ is any other imbedding of $\mathfrak{R}$, then there exists an associative homomorphism of $\mathfrak{I}^{*}$ onto the enveloping algebra of $\sigma$. A universal imbedding of a finite-dimensional Lie algebra $\&$ with basis $x_{1}, \cdots, x_{n}$ may be constructed as follows. Form the free associative algebra $\mathfrak{F}$ over $K$ on the elements $x_{1}, \cdots, x_{n}$, and consider the difference algebra $\mathfrak{F} / \mathfrak{B}$, where $\mathfrak{B}$ is the ideal generated by the elements $x_{i} x_{j}-x_{j} x_{i}-\sum \gamma_{i j k} x_{k}$, and $\left[x_{i} x_{j}\right]=\sum \gamma_{i j k} x_{k}$ is the multiplication table in $\mathbb{R}$. Then the mapping $\eta: \sum \alpha_{i} x_{i} \rightarrow \sum \alpha_{i}$ $\cdot\left(x_{i}+\mathfrak{B}\right)$ of $\mathfrak{R}$ into $\mathfrak{I}^{*}$ is a universal imbedding, and $\mathfrak{A}^{*}$ is the enveloping algebra of $\eta$. If we denote the cosets of the $x_{i}$ by $\bar{x}_{i}$, then it can be proved that the standard monomials $\bar{x}_{1}^{e_{1}^{1}} \cdots \bar{x}_{n}^{e_{n}},\left(e_{1}, \cdots, e_{n}\right) \neq 0$, form a basis of $\mathfrak{A}^{*}$ over $K$, and that, since the $\bar{x}_{i}$ are linearly independent, the imbedding $\eta$ is faithful. We shall identify $\&$ with the linear part of $\mathfrak{A}^{*}$, and in particular, write $x_{1}, \cdots, x_{n}$ for the $\bar{x}_{i}$.

The importance of the universal enveloping algebra $\mathfrak{A}^{*}$ for the theory of representations stems from the fact that there is a natural one-to-one correspondence between the representations of $\mathfrak{R}$ and the representations of $\mathfrak{A}^{*}$, which can be described as follows. If $\sigma$ is a representation ${ }^{2}$ of $\mathbb{R}$, then the unique associative homomorphism of $\mathfrak{A}^{*}$ onto the enveloping algebra of $\sigma$ defines a representation of $\mathfrak{H}^{*}$, and conversely, if $\tau$ is a representation of $\mathfrak{2}^{*}$, and if $\eta$ is the universal imbedding, then $\sigma=\eta \tau$ is a representation of $\&$.

For various technical reasons it is convenient to adjoin an identity element $1 \equiv x_{1}^{0} x_{2}^{0} \cdots x_{n}^{0}$ to $\mathfrak{A}^{*}$, and the algebra $\mathfrak{A}$ obtained, while it is not, strictly speaking, a universal enveloping algebra of $\mathbb{R}$, will be called nevertheless the universal associative algebra of $\mathbb{R}$. The one-toone correspondence between the representations of $\mathbb{R}$ and those of $\mathfrak{A}$ persists, for we can extend the representation of $\mathfrak{A}^{*}$ corresponding to a given representation of $\mathfrak{R}$ to one of $\mathfrak{A}$ by mapping the identity element of $\mathfrak{A}$ onto the identity linear transformation.

We now introduce an increasing filtration of $\mathfrak{A}$, that is, a family $\left\{\mathfrak{A}_{i},-\infty<i<\infty\right\}$ of subspaces of $\mathfrak{A}$ such that $\mathfrak{H}_{i} \subseteq \mathfrak{A}_{i+1}, \cup \mathfrak{H}_{i}=\mathfrak{A}$, and $\mathfrak{H}_{i} \mathfrak{A}_{j} \subseteq \mathfrak{A}_{i+j}$. In fact, we set $\mathfrak{A}_{-1}=\mathfrak{A}_{-2}=\ldots=(0) ; \mathfrak{H}_{0}=K \cdot 1$, $\mathfrak{H}_{1}=\mathfrak{R}$, and we define $\mathfrak{H}_{k}, k>1$, inductively to be the subspace $\mathfrak{A}_{k-1}$ $+\Omega^{k}$, where $\mathfrak{R}^{k}$ consists of all finite sums of products $\alpha u_{1} u_{2} \cdots u_{k}$, $\alpha \in K, u_{i} \in \mathbb{R}$. Then we define the degree $d(x)$ of an element $x$ by the formula $d(x)=\min _{x \in \mathfrak{N} i} i$; then $d$ is a function on $\mathfrak{A}$ to the non-negative integers and $-\infty$ such that (i) $d(\alpha x) \leqq d(x), \alpha \in K$, (ii) $d(x+y)$ $\leqq \max (d(x), d(y))$, and (iii) $d(x y) \leqq d(x)+d(y)$. This definition is independent of the choice of a basis in $\mathfrak{A}$, but it can be shown easily that $d(x)$ coincides with the notion of degree one is led to by writing

\footnotetext{
${ }^{2}$ It is not assumed that the representation space is finite-dimensional.
} 
out $x$ as a linear combination of the standard monomials.

Corresponding to the algebra $\mathfrak{A}$, together with the filtration $\left\{\mathfrak{U}_{i}\right\}$, we can associate a graded algebra $\mathfrak{M}$ which is, as a vector space, the direct sum of the spaces $\mathfrak{A}_{i} / \mathfrak{A}_{i-1}$, and where the product of two cosets $\bar{s}_{i} \in \mathfrak{A}_{i} / \mathfrak{A}_{i-1}$ and $\bar{s}_{j} \in \mathfrak{A}_{j} / \mathfrak{A}_{j-1}$ is defined to be the coset in $\mathfrak{A}_{i+j} / \mathfrak{A}_{i+j-1}$ of $s_{i} s_{j}$, where $s_{i} \in \bar{s}_{i}$, and $s_{j} \in \bar{s}_{j}$. The following result is the key to many of the arithmetic properties of the algebra $\mathfrak{A}$.

Theorem 1. The graded algebra $\mathfrak{M}$ associated with the universal associative algebra $\mathfrak{A}$ of a Lie algebra $\mathfrak{R}$, together with its filtration by degrees, is isomorphic to the polynomial ring $K\left[X_{1}, \cdots, X_{n}\right]$, where $n$ is the dimension of $\mathbb{R}$.

This result seems to provide the natural setting for the following properties of the algebra $\mathfrak{A}$, which can also be proved directly. The first result of interest states that the function $d$ satisfies a sharper form of the relation (iii) above, namely $d(x y)=d(x)+d(y)$. This formula implies on the one hand that $\mathfrak{A}$ has no divisors of zero, and on the other hand, that the units of $\mathfrak{A}$ coincide with the nonzero elements of $K \cdot 1$, so that $\mathfrak{A}$ is semi-simple in the sense of Jacobson.

A second property of $\mathfrak{A}$, first established by Birkhoff and Whitman [2], states that $\mathfrak{A}$ satisfies the maximum condition for left ideals.

We assume now, and throughout the remainder of the article, that the base field $K$ has characteristic $p>0$.

$\mathrm{N}$. Jacobson proved in [7] that for each generator $x_{i}$ of $\mathfrak{A}$ there exists a nonzero polynomial $f_{i} \in K[\lambda]$ such that $y_{i}=f_{i}\left(x_{i}\right)$ is in the center $\mathfrak{C}$ of $\mathfrak{A}$. Let $\Re=K\left[y_{1}, \cdots, y_{n}\right]$. Then $\Re$ has two properties of importance: the commuting elements $y_{i}$ are algebraically independent over $K$, so that $\Re$ is isomorphic to the polynomial ring in $n$ variables; and it is possible to find a finite $\Re$-linearly independent set of $p^{N}$ generators for the $\Re$-module $\mathfrak{U}$, where $p^{N}$ is the product of the degrees of the polynomials $y_{i}$. Now let $R$ be the quotient field of $\Re$, and form the tensor product $D=\mathfrak{A} \otimes R$ with respect to $\mathfrak{R}$. Since $\mathfrak{A}$ is a free $\mathfrak{R}$-module, the natural mapping $x \rightarrow x \otimes 1$ of $\mathfrak{A}$ into $\mathfrak{A} \otimes R$ is an isomorphism of $\mathfrak{A}$ into $D$, and we shall identify $\mathfrak{A}$ with its image in $D$. Then $D$ has no divisors of zero, and since $D$ is a finite-dimensional algebra over $R, D$ is a division algebra. The center of $D$ is the quotient field $C$ of $\mathfrak{E}$, the center of $\mathfrak{A}$, and the dimension of $D$ over $C$ is an even power of $p$, which we shall denote by $p^{2 m}$. Finally it can be proved that $\mathfrak{A}$ is a bounded maximal $\operatorname{order}^{3}$ in $D$, that $\mathfrak{S}$ is integrally closed in $C$, and that $\mathfrak{E}$ is integrally dependent on $\Re$ so that $\mathfrak{E}$ has degree of transcendence $n$ over $K$, where $n$ is the dimension of $\mathbb{R}$.

${ }^{8}$ Cf. N. Jacobson, The theory of rings, New York, 1943, Chap. 6. 
3. General properties of the representations. We consider first an arbitrary irreducible representation $\sigma$ of $\mathfrak{A}$ with kernel $\mathfrak{U}$. It is proved in [4] that $\mathfrak{A} / \mathfrak{U}$ is a finite-dimensional algebra over $K$, and this implies that every irreducible representation of $\mathfrak{A}$ (and hence of $\mathfrak{R}$ ) has finite degree. If we combine this fact with the result that $\mathfrak{A}$ is a semisimple algebra, so that the intersection of the kernels of the irreducible representations of $\mathfrak{A}$ is the zero ideal, then we can prove that $\mathfrak{A}$ has sufficiently many (finite-dimensional) irreducible representations. A consequence of this result is the following theorem (cf. [4]), which is actually a sharpened form of a result proved by Iwasawa [5] and Jacobson [7].

THEOREM 2. Every finite-dimensional Lie algebra over a field of characteristic $p>0$ has a faithful finite-dimensional completely reducible representation.

The rift between characteristic $p$ and characteristic 0 widens when one observes that there is no class of algebras of characteristic $p$ every one of whose representations is completely reducible. The precise result, which was conjectured by Chevalley and proved by Jacobson [7], is the following.

THEOREM 3. Every finite-dimensional Lie algebra over a field of prime characteristic has a finite-dimensional representation which is not completely reducible.

To gain a deeper insight towards the behavior of the irreducible representations, we adopt the approach of $\mathrm{H}$. Zassenhaus, and consider the relations among the representations of the center $\mathbb{C}$ of $\mathfrak{A}$ and the representations of $\mathfrak{A}$ itself. A homomorphism $\Phi$ of $\mathfrak{U}$ is said to be an extension of a homomorphism $\phi$ of $\mathbb{C}$ if $\phi$ is the restriction of $\Phi$ to $\mathfrak{E}$. We note that if $\Phi$ extends $\phi$, then the image $\mathfrak{A} \Phi$ of $\mathfrak{A}$ is a $\mathfrak{C} \phi$ module. Then we can state a theorem due to Zassenhaus, which asserts that if $\phi$ is a homomorphism of $\mathbb{E}$ onto the base field $K$, then there exists a generic extension $\Phi$ of $\phi$ with the property that if $\Psi$ is any other extension of $\phi$, then there exists a $\ \phi$-module homomorphism $\tau$ of $\mathfrak{2} \Phi$ onto $\mathfrak{R} \Psi$. The proof of this result depends only upon the structure of $\mathfrak{A}$ as a finitely generated $\mathfrak{C}$-module, and is based on the theory of elementary ideals due to Steinitz and Zassenhaus. ${ }^{4}$

The main results on the absolutely irreducible representations which have been obtained to date, and which are due to Zassenhaus, can be derived in two ways: either by making use of the minimum polynomial of $\mathfrak{A}$ as Zassenhaus has done, or by applying certain re-

' Cf. H. Zassenhaus, Lehrbuch der Gruppentheorie, Leipzig, 1937. 
sults on minimal identities for matrix algebras due to Levitzki and Amitsur [8]. We shall indicate both approaches to the subject, because the relationship between them is a problem still to be worked out.

First we consider Zassenhaus' approach. We select a module basis $a_{1}, \cdots, a_{r}$ for $\mathfrak{A}$ over $\mathfrak{E}$, and define the general element $a$ of $\mathfrak{A}$ to be $\sum_{i=1}^{r} X_{i} a_{i}$, an element of the ring $\mathfrak{A}_{\mathfrak{B}}$ obtained by extending the coefficient ring of $\mathfrak{A}$ from $\mathfrak{C}$ to $\mathfrak{P}=\mathfrak{C}\left[X_{1}, \cdots, X_{r}\right]$, where the $X_{i}$ are algebraically independent over $\mathfrak{E}$. Then $A_{\Re}$ is a subring of the algebra $D_{Q}$ obtained by extending the coefficient field of $D$ from the quotient field of $\mathbb{S}$ to $Q$, the quotient field of $\mathfrak{B}$, and $a$ is an element of the finite-dimensional algebra $D_{Q}$. Then $a$ has a minimum polynomial $f(\lambda)$, whose coefficients are in $\mathfrak{F}$ since $\mathfrak{E}$ is integrally closed.

By comparing the degree of $f(\lambda)$ with the degree of the minimum polynomial of the general element of $A \Psi$, where $\Psi$ is an a.i.r. of $A$, the following result is obtained.

THEOREM 4. The degree of every absolutely irreducible representation of $\mathfrak{A}$ is less than or equal to $p^{m}$, where $p^{2 m}$ is the dimension of $D$ over its center.

The trace of the general element $a$ of $\mathfrak{A}$, which is the negative of the second coefficient of $f(\lambda)$, has the form $\sum_{i=1}^{r} \lambda_{i} X_{i}, \lambda_{i} \in \mathbb{E}$. We then define the reduced trace $\operatorname{tr}(u)$ of an arbitrary element $u \in \mathfrak{A}, u=\sum \xi_{i} a_{i}$, $\xi_{i} \in \mathfrak{S}$, by the formula $\operatorname{tr}(u)=\sum \lambda_{i} \xi_{i}$. Corresponding to the trace function, there is a bilinear form $g$ on $\mathfrak{U} \times \mathfrak{U} \rightarrow \mathfrak{C}$, namely $g(x, y)=\operatorname{tr}(x y)$. We define the discriminant $\mathfrak{b}$ of $\mathfrak{A}$ over $\mathfrak{S}$ to be the ideal in $\mathfrak{S}$ generated by the determinants $\left|g\left(u_{i}, v_{k}\right)\right|$, where $u_{1}, \cdots, u_{p}{ }^{2 m}$ and $v_{1}, \cdots, v_{p}^{2 m}$ are arbitrary elements of $\mathfrak{A}$. Then we can state

TheOREM 5. Let $K$ be an algebraically closed field. Let $\psi$ be a homomorphism of $\mathbb{S}$ onto $K$, and let $\Psi$ be a generic extension of $\psi$. Then the following statements are equivalent.

(i) $\mathfrak{R} \Psi$ is a central simple algebra over $K$;

(ii) $\mathfrak{H} \Psi$ has an irreducible representation of degree $p^{m}$, and hence every irreducible representation extending $\psi$ has degree $p^{m}$;

(iii) $\delta \psi \neq(0)$.

The bound on the degrees of the a.i.r. of $\mathfrak{A}$ can also be obtained by applying the result of Levitzki and Amitsur, which states that the standard identity $S_{2 n}(x)$ in $2 n$ variables vanishes identically on the full matrix algebra of order $n$, but not on the full matrix ring of order $s$ for $s>n$. Moreover, if we consider the ideal $\tilde{\delta}$ in $\mathfrak{A}$ generated by the elements $S_{2\left(p^{2 m}-1\right)}\left(a_{1}, \cdots, a_{2\left(p^{2 m}-1\right)}\right)$ where the $a_{i}$ are arbitrary in $\mathfrak{A}$, 
then a result analogous to Theorem 5 can be proved, specifically that the degree of an a.i.r. $\Psi$ is $p^{m}$ if and only if $\tilde{\mathfrak{D}} \Psi \neq(0)$.

The following results have been obtained by Zassenhaus concerning the indecomposable representations.

Theorem 6. Let $K$ be algebraically closed, and let $\Psi$ be an indecomposable representation of $\mathfrak{A}$. Then any two irreducible constituents of $\Psi$ have the same restrictions to $\mathbb{C}$.

There are examples, however, which show that an indecomposable representation may have inequivalent irreducible constituents.

THEOREM 7. Let $K$ be algebraically closed, and let $\psi$ be an arbitrary homomorphism of $\mathfrak{E}$ onto $K$. Then there exist indecomposable representations of arbitrarily high degree, each of which has an irreducible constituent extending $\psi$.

4. Special results and problems. The representation theory of nilpotent Lie algebras over an algebraically closed field of characteristic $p>0$ has been studied fully by Zassenhaus in [10] and [11]. We summarize his main results, some of which (in particular Theorem 8) have been extended to nilpotent Lie algebras over an infinite field by N. Jacobson and the writer.

THEOREM 8. If $\Psi$ is an indecomposable representation of a nilpotent Lie algebra \& over an algebraically closed field, then any two irreducible constituents of $\Psi$ are equivalent.

THEOREM 9. Let $\&$ be a nilpotent Lie algebra over an algebraically closed field $K$. Then the degree of any irreducible representation of $\mathbb{R}$ is a power of $p$. If we select in \& a basis $\left(a_{1}, \cdots, a_{n}\right)$ such that, whenever $i<j,\left[a_{i} a_{j}\right] \in\left(a_{i+1}, \cdots, a_{n}\right)$, then for each ordered set $\left(\lambda_{1}, \cdots, \lambda_{n}\right)$ of elements of $K$, there exists one and only one irreducible representation $\Gamma: x \rightarrow x \Gamma$ of $\mathfrak{R}$ such that $\lambda_{i}$ is the unique eigenvalue of $a_{i} \Gamma, i=1, \cdots, n$.

A determination of the irreducible representations of the Witt algebras has been given by Ho-Jui Chang in [3]. By making use of graded algebra techniques, Zassenhaus has given new proofs of a number of these results [12]; in particular he has given a direct proof of the theorem of Chang which states that the integer $m$ for the $p$-dimensional Witt algebra $\mathbb{R}$ is exactly $(p-1) / 2$, and thus the bound on the degree of the absolutely irreducible representations of $\mathbb{R}$ is given explicitly in this case. The values of $m$ for simple algebras other than the Witt algebras are unknown at this time.

We know that in general the center $\mathfrak{C}$ of $\mathfrak{A}$ does not coincide with the ring $\Re=K\left[y_{1}, \cdots, y_{n}\right]$ described above. It seems to be an inter- 
esting problem to determine the structure of the field extension of the quotient field of $\mathfrak{C}$ over the quotient field of $\Re$. It is known that for nilpotent Lie algebras the extension is purely inseparable.

The existence of an extension to $\mathfrak{A}$ of a given homomorphism $\psi$ of $\mathfrak{E}$ is known only when $\psi$ maps $\mathbb{E}$ onto a field. For the study of the indecomposable representations it is of importance to know whether $\psi$ can be extended if $\mathbb{S} \psi$ is a primary algebra. Progress in this direction may wait upon a knowledge of the ideal theoretic properties of $\mathfrak{A}$ considered as a maximal order in $D$.

Jacobson has proved in [6] that a restricted Lie algebra $\&$ has only a finite number of inequivalent irreducible representations. The representations of $\mathfrak{R}$ are in $1-1$ correspondence with the representations of the $\mathfrak{U}$-algebra of $\mathfrak{R}$, which is a finite-dimensional algebra. The problem of the determination of the representations of $\mathbb{R}$ involves first of all detailed information concerning the radical of the $\mathfrak{U}$-algebra of $\mathfrak{R}$.

Another problem concerns the construction of representations of a Lie algebra $\&$ having prescribed constituents. For example, if $\Psi_{1}$ and $\Psi_{2}$ are irreducible representations of $\mathfrak{R}$, does there exist an indecomposable representation of $\mathbb{R}$ having $\Psi_{1}$ and $\Psi_{2}$ as constituents? This problem can be formulated in terms of a cohomology theory for representation spaces.

\section{REFERENCES}

1. G. Birkhoff, Representability of Lie algebras and Lie groups by matrices, Ann. of Math. vol. 38 (1937) pp. 326-332.

2. G. Birkhoff and P. Whitman, Representations of Jordan and Lie algebras, Trans. Amer. Math. Soc. vol. 65 (1949) pp. 116-136.

3. Ho-Jui Chang, Über Wittsche Lie-Range, Abh. Math. Sem. Hansischen Univ. vol. 14 (1941) pp. 151-184.

4. C. W. Curtis, Non-commutative extensions of Hilbert rings, Proc. Amer. Math. Soc., to appear.

5. K. Iwasawa, On the representation of Lie algebras, Jap. J. Math. vol. 19 (1948) pp. 405-426.

6. N. Jacobson, Restricted Lie algebras of characteristic p, Trans. Amer. Math. Soc. vol. 50 (1941) pp. 15-25.

7. - A note on Lie algebras of characteristic p, Amer. J. Math. vol. 74 (1952) pp. 357-359.

8. J. Levitzki and S. Amitsur, Minimal identities for algebras, Proc. Amer. Math. Soc. vol. 1 (1950) pp. 449-463.

9. E. Witt, Treue Darstellung Liescher Ringe, J. Reine Angew. Math. vol. 176 (1937) pp. 126-140.

10. H. Zassenhaus, Über Liesche Ringe mit Primzahlcharacteristik, Abh. Math. Sem. Hansischen Univ. vol. 13 (1940) pp. 1-100.

11. - Darstellungstheorie nilpotenter Lie-Ring bei characteristic $p>0, \mathrm{~J}$. Reine Angew. Math. vol. 182 (1940) pp. 150-155. 
12. - Representation theory of Lie algebras of prime characteristic, Mimeographed notes, Summer Mathematical Institute, Waterville, 1953.

C. W. Curtis

\section{SEMINAR ON SIMPLE LIE ALGEBRAS}

The heart of the structure theory for Lie algebras of characteristic 0 is given by the following two statements: (1) An algebra with no solvable ideals is a direct sum of simple algebras, (2) The simple algebras are given by an explicit list, comprising four infinite families and five exceptional algebras. Statement (1) becomes false for characteristic $p$, and finding a substitute for it is a problem that awaits solution. It seems to be timely to attack the classification of simple Lie algebras of characteristic $p$. Very likely a complete solution of this second problem would carry with it valuable hints for the solution of the first. It is our purpose to give a list (believed to be essentially complete) of the known simple Lie algebras over an algebraically closed field. The list naturally falls into three parts: the classical algebras, the exceptional algebras, and the Witt algebra and its generalizations.

Classical algebras. Type A. Let $T$ be the Lie algebra of all $n$ by $n$ $(n \geqq 2)$ matrices of trace 0 over a field $F$. If the characteristic of $F$ is 0 or prime to $n, T$ is a simple algebra (of dimension $n^{2}-1$ ). If the characteristic does divide $n, T$ still has a one-dimensional center $Z$ consisting of all scalar matrices. Except when the characteristic is two and $n=2, T / Z$ is simple and has dimension $n^{2}-2$.

Types B, D. A matrix $\left(a_{i j}\right)$ is alternate if $a_{i i}=0, a_{i j}=-a_{j i}$ for all $i$ and $j$. Let $S$ be the Lie algebra of all alternate $n$ by $n$ matrices over a. field. For $n=3$ and $n \geqq 5, S$ is a simple algebra of dimension $n(n-1) / 2$.

Type C. On a two-by-two matrix algebra we may define an involution ${ }^{*}$ as follows:

$$
\left(\begin{array}{ll}
a & b \\
c & d
\end{array}\right) \rightarrow\left(\begin{array}{rr}
d & -b \\
-c & a
\end{array}\right) .
$$

A matrix of even order, split into two by two blocks as $\left(A_{i j}\right)$, admits an extension of this involution: $\left(A_{i j}\right)^{*}=\left(A_{j i}^{*}\right)$. Let $S$ denote the Lie algebra of all matrices of order $2 m$, skew with respect to this involution; $S$ has dimension $2 m^{2}+m$. For characteristic different from two, $S$ is simple as it stands. If the characteristic is two and $m$ is odd, $m \geqq 3$, the cube of $S$ is a simple algebra of order $2 m^{2}-m-1$. If $m$ is even, $m \geqq 4$, the cube of $S$ still has to be reduced modulo a one-dimensional center, and we get a simple algebra of order $2 m^{2}-m-2$. 
Exceptional algebras. Let $C$ be the Cayley matrix algebra (i.e., the unique Cayley-Dickson algebra with no divisors of zero) over a field $F$. Let $A$ be the algebra of derivations of $C$. Then $A$ is always 14-dimensional, and for characteristic different from 3 it is simple. When the characteristic is 3, $A$ acquires a 7-dimensional ideal $I$ of inner derivations, and $I$ is isomorphic to the algebra of 3 by 3 matrices of trace 0 , reduced modulo the center. The algebra $A / I$ of outer automorphisms of $I$ is again isomorphic to $I$.

For characteristic 0 there are four more exceptional algebras, of orders $52,78,133$, and 248. The analogues of these for characteristic $p$ have yet to be investigated. Schafer and Tomber have proved that the obvious candidate for the 52-dimensional one (the derivations of the exceptional Jordan algebra) degenerates for characteristic two by acquiring a 26-dimensional ideal of inner derivations.

The Witt algebra and its generalizations. Let $F$ be a field of characteristic $p$ and $B$ the (commutative associative) $p$-dimensional algebra over $F$ generated by an element $x$ satisfying $x^{p}=0$. Let $A$ be the algebra of derivations of $B$. If we write $D_{i}$ for the derivation sending $x$ into $x^{i+1}$, the $D$ 's form a basis of $A$ with multiplication table $\left[D_{i} D_{j}\right]=(i-j) D_{i+j} . B$ is a $p$-dimensional Lie algebra which is simple for $p \neq 2$; it was discovered by Witt.

Two generalizations have been given in the literature. Zassenhaus used the same multiplication table, with the subscripts allowed to range over any additive subgroup of $F$ (instead of the integers $\bmod p$ ). Jacobson took the derivation algebra of the algebra generated by several indeterminates with $p$ th power 0 .

There is a family of algebras which includes both of these general izations. Let $V$ be a vector space over $F$, and $G$ a total additive group of functionals on $V$. Let $A$ be the vector space direct sum of copies of $V$, one for each member of $G$. The general element of $A$ is expressible as a sum over $G$ of elements $(x, \alpha), x \in V, \alpha \in G$. We define

$$
[(x, \alpha)(y, \beta)]=(\alpha(y) x-\beta(x) y, \alpha+\beta) .
$$

The resulting Lie algebra is simple except when $V$ is one-dimensional and the characteristic is two; there is then a simple ideal with dimension smaller by one. Zassenhaus' algebra is the case where $V$ is onedimensional, while Jacobson's is the case where $G$ consists of all integer-valued functionals.

I. KAPLANSKY 